Brit. Heart J., 1964, 26, 755.

\title{
RIGHT AND LEFT HEART BIPLANE CINE-ANGIOCARDIOGRAPHY
}

BY

\author{
HAMISH WATSON AND C. PICKARD
}

From the Departments of Medicine and Diagnostic Radiology in the University of St. Andrews, Queen's College, Dundee, and the Dundee Royat Infirmary

Received November 15, 1963

Technological progress in the design and construction of image intensifiers has been so rapid since their introduction by Teves (1955) and Feddema (1955) that they are already in daily use for many types of radiological examination. Though much of the pioneer work in this field was angiocardiographic (Lind, Wegelius, and Lichtenstein, 1954; Lind, Wegelius, and Boesen, 1955; Lind, Rocha, and Wegelius, 1957; Stauffer et al., 1955; Astley, 1955, 1956; Pickard and Watson, 1957; Watson et al., 1958), there were certain disadvantages associated with the use of the early intensifiers for this type of investigation. The 5-inch field, for example, placed considerable limitation on their usefulness, and this, coupled with the fact that it was only possible to film in one plane, meant that the procedure had to be carefully planned not only to decide which part of the heart was to be visualized but also how best to position the patient to ensure that its opacification would be seen to the best possible advantage. Despite the difficulties, cine-photography of the intensified image has already revolutionized the visualization of the opacified heart and contributed greatly not only to our understanding of valvular action but also to our knowledge of the intracardiac circulation in congenital heart disease.

The field size of intensifiers has been increased during the past few years and a much larger image can now be obtained. The definition of detail has been greatly improved and the recent addition of closed circuit television allows the clearer pictures to be much more easily seen. The object of this paper is to present a preliminary communication on our experience of simultaneous biplane cine-angiocardiography using two 9-inch Philips image intensifiers.

\section{METHOD}

Both intensifiers are fitted with television and cine-cameras. The units are placed at right angles as shown in Fig. 1, and the two systems are entirely separate. The x-ray generators are activated electrically by impulses arriving from the cameras and, since the $\mathrm{x}$-ray emissions are individual pulses, it is possible to control the exposure of each intensifier unit over a very wide range. The two generators fire out of phase and this sequence is maintained by a very efficient electrical link using the "mag slip" principle. The speed of each cine-camera is at present limited to 50 frames a second. As each intensifier has a built-in television camera as well as a $35 \mathrm{~mm}$. Arriflex cine-camera, television monitoring is possible in either plane or in both planes simultaneously during placement of the catheter for selective injections, and biplane viewing is continued in a modified form throughout the cine exposure so that the angiocardiogram may be watched as it takes place. It is thus possible to make an immediate assessment of the gross structural anatomy and of the quality of its opacification, and to decide whether or not further injections of contrast medium are required. If there is any doubt, the procedure is suspended for about 20 minutes and the dried films are examined as they come off the automatic processor.

$3 \mathrm{E}$ 


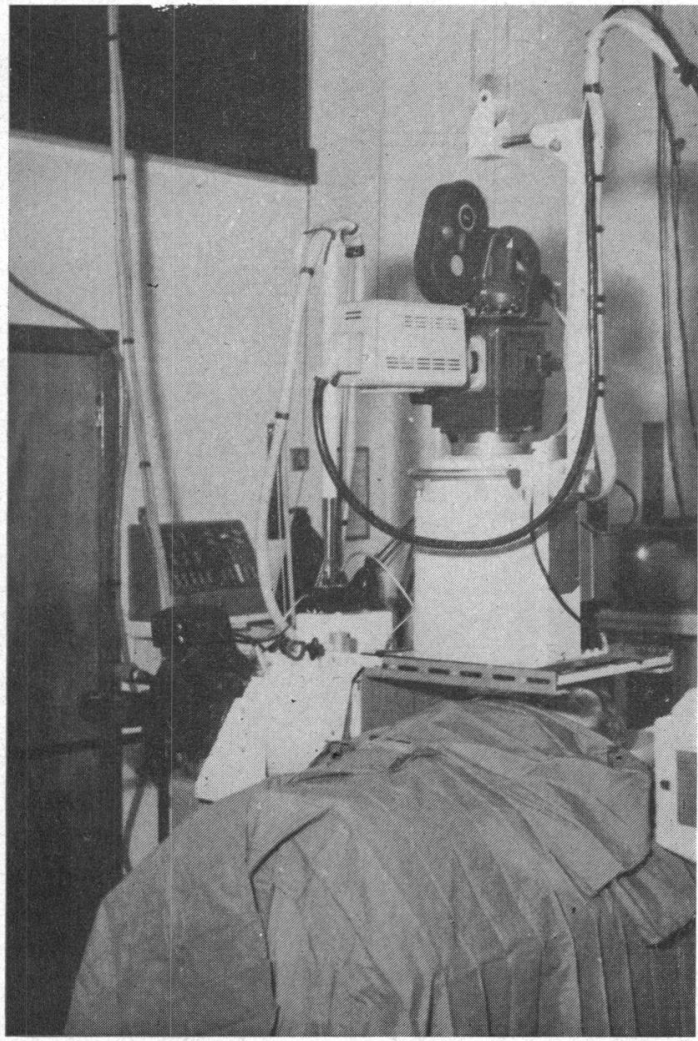

Fig. 1.-The two 9-inch image intensifiers, each with television and cine-cameras, mounted at right angles.
Except in special circumstances, our current practice is to make a selective injection into the right ventricle, watch and film it in two planes, and then to repeat the process by injecting into the left atrium.

We have found Triosil a safe and satisfactory preparation especially suited to babies and small children because of its low viscosity, and use 1.0 to $1.5 \mathrm{ml}$. per kilogram of body weight for each injection. The volume required depends on individual circumstances, varying with the magnitude and direction of intracardiac shunts and the severity of closed chamber stenoses. As a general rule satisfactory opacification of the left heart is obtained by using less contrast medium than is required for the right, though this probably reflects only the difference between atrial and ventricular injections. Fig. 2 and 3 show excerpts of posteroanterior and lateral cine-films taken simultaneously at 50 frames a second during the investigation of a 3-year-old girl with Fallot's tetralogy. In Fig. 2, opacification of both the pulmonary trunk and the aorta are seen following an injection of $18 \mathrm{ml}$. of 75 per cent Triosil into the apex of the right ventricle. The severe nature of the outflow tract obstruction is revealed in the lateral film where the infundibulum is seen to be grossly narrowed over its whole length. It is of interest to note the poststenotic dilatation of the pulmonary trunk, which is almost as wide as the dilated overriding aorta.

Fig. 3 demonstrates the left heart opacification after injection of a further $12 \mathrm{ml}$. of Triosil into the left atrium which was entered through a valve-patent foramen ovale. This confirms the presence of a left ventricle and that it gives rise to the dextroposed aorta; in addition it confirms the virtual absence of left-to-right shunting in this case.

\section{DISCUSSION}

This new equipment obviates many of the difficulties that were associated with the use of small single plane image intensifiers. Our early experience with simultaneous biplane cine-angiocardiography of the right and left heart has been encouraging. Following cardiac catheterization this method of investigation seldom leaves one short of essential information and we have found it particularly well suited to children with serious congenital heart defects in the first year of life and in the newborn period. Since an important proportion of current infant mortality results from such lesions, we have, for some time now, concentrated much of our effort on this age-group, believing that surgical emphasis will be increasingly directed towards reducing this wastage of infant life.

With present resources a very complete diagnostic investigation can be carried out on such cases, but much better facilities must be developed for the analysis of cine-angiocardiographic records. The problem of viewing is an acute one for there is as yet no easy way of extracting the wealth of information that is available on the cine-films. It is, of course, part of the somewhat difficult transition from standard radiology with large single films, which everyone is equipped to view, to the new rapidly spreading radiology with image intensifiers, television cameras, and cinefilms which no one is properly equipped to view. The time is rapidly approaching when a small projection room will be required in all large departments of diagnostic radiology, where cardiologist 


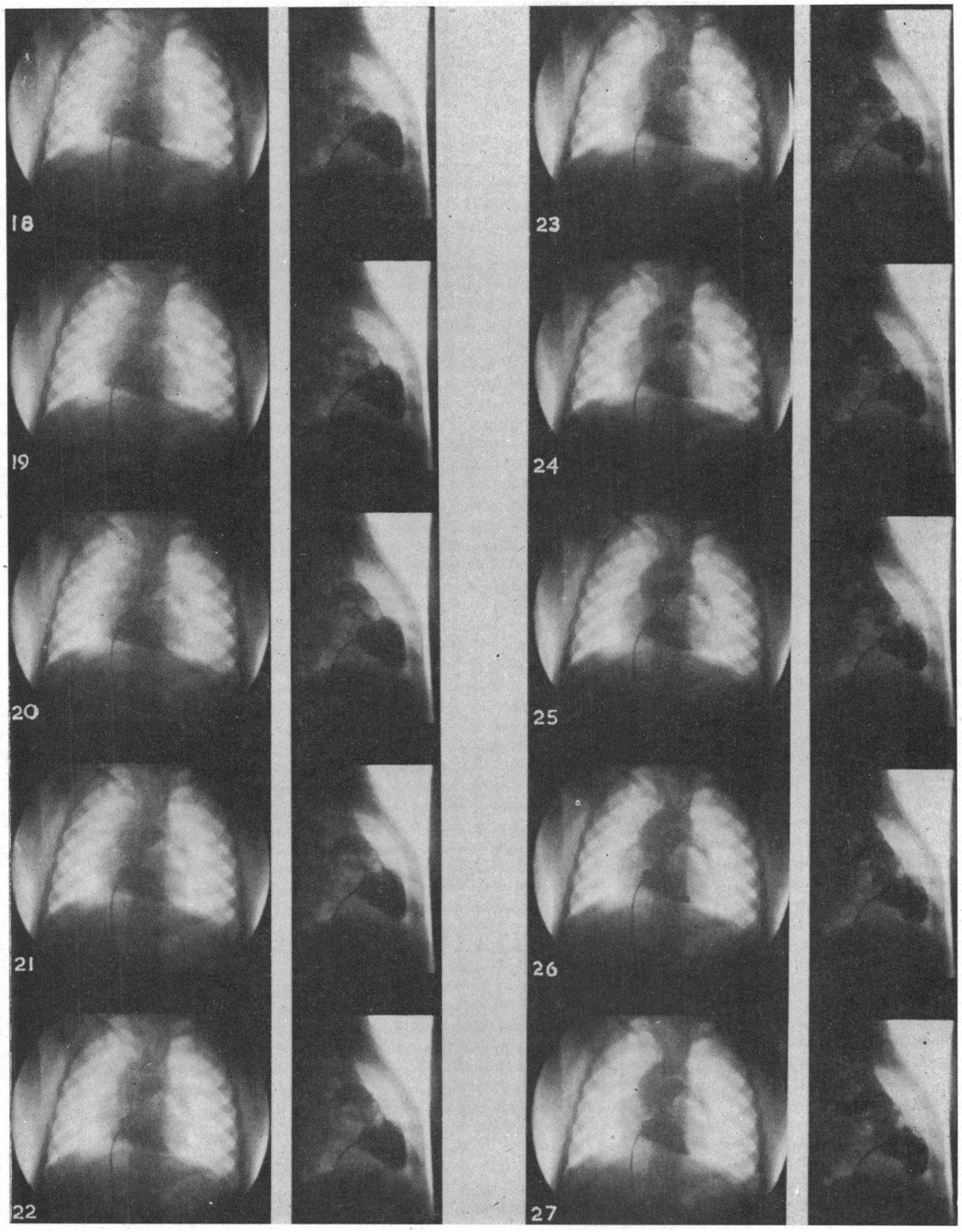

Fig. 2.-A series of 10 consecutive frames, excerpted from simultaneously exposed postero-anterior and lateral films taken at 50 frames a second, following a right ventricular injection of $18 \mathrm{ml}$. of 75 per cent Triosil in a 3-year-old girl with Fallot's tetralogy.

and radiologist can watch cine-films together at varying speeds and as serial stills. The handling of this material demands the services of a professional photographer, who will be an essential member of staff in charge of processing, projection, and production of enlarged stills selected for further study.

The introduction of biplane television screening has made it very much easier to demonstrate 


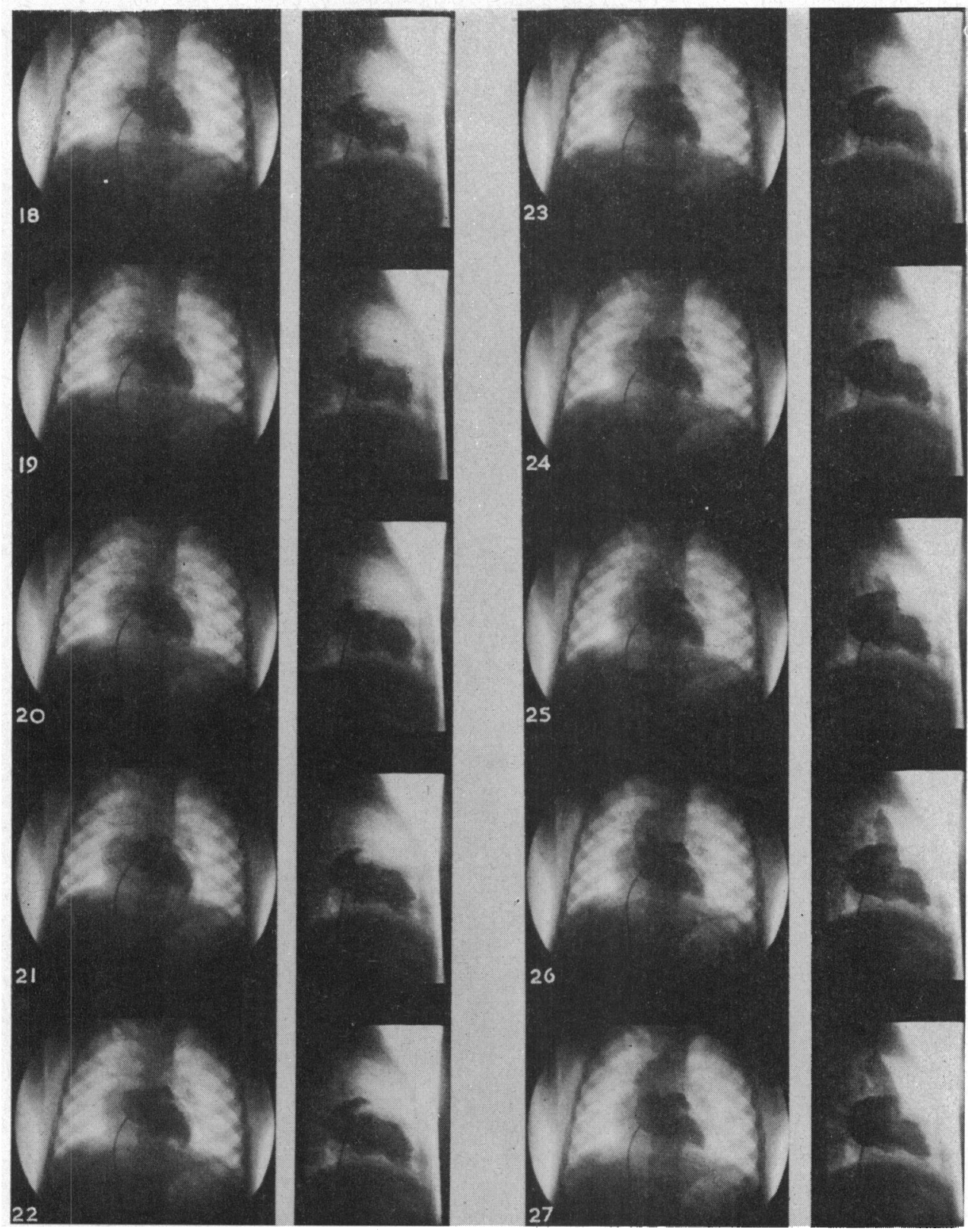

FIG. 3.-A series of 10 consecutive frames, excerpted from simultaneously exposed postero-anterior and lateral films taken at 50 frames a second, following a left atrial injection of a further $12 \mathrm{ml}$. of 75 per cent Triosil in the same patient as in Fig. 2.

cardiac catheterization and angiocardiography to an audience, and this is a valuable aid to postgraduate teaching. Furthermore, it makes these procedures much less tedious for the many essential assistants who, in the past, have had to stand about for long hours in the dark seeing little or nothing of what was actually happening.

A great deal remains to be learned about the intracardiac circulation, the movement of valves, 
and the details of pulmonary blood flow in all types of heart disease. Though this method promises well for their future study, much better methods must be developed to deliver contrast medium at desired sites in the heart without producing arrhythmias, and accurate electrocardiographic correlation with phasic injection is essential if its value is to be fully exploited.

\section{SUMMARY}

Right and left heart biplane cine-angiocardiography using 9-inch image intensifiers and closed circuit television is described and illustrated.

The value of this technique in the diagnosis of severe congenital heart disease in the first year of life is stressed.

Problems associated with viewing and the analysis of films are discussed.

\section{REFERENCES}

Astley, R. (1955). Cineradiography with an image amplifier: a practical technique. Brit. J. Radiol., n.s., $28,221$. (1956). Cineangiocardiography. Brit. J. Radiol., n.s., 29, 556.

Feddema, J. (1955). Some possible diagnostic applications of cineradiography. Proceedings of Annual Congress, British Institute of Radiology, 1953. Brit. J. Radiol., n.s., 28, 217.

Lind, J., Rocha, M., and Wegelius, C. (1957). The value of fast angiocardiography in the early diagnosis of patent ductus arteriosus. Amer. J. Roentgenol., 77, 235.

- Wegelius, C., and Boesen, I. (1955). Recent developments in angiocardiography. In Henry Ford Hospital International Symposium on Cardiovascular Surgery, ed. R. C. Larn, p. 30. Saunders, Philadelphia.

- , and Lichtenstein, H. (1954). The dynamics of the heart in complete A-V block. Circulation, $10,195$.

Pickard, C., and Watson, H. (1957). Selective cine-angiocardiography using image intensification. In Proceedings of the British Cardiac Society, Annual General Meeting. Brit. Heart J., 19, 585.

Stauffer, H. M., Morton, J., Oppenheimer, M. J., Stewart G. H., III, and Blackstone, A. W. (1955). Practical image amplifier technics; fluoroscopy, cinefluorography, spot-film radiography and use with closed circuit television. Radiology, 65, 784 .

Teves, M. C. (1955). Image intensification. Read at the Annual Congress of the British Institute of Radiology, December, 1953. Brit. J. Radiol., n.s., 28, 216.

Watson, H., Pickard, C., Lowe, K. G., and Hill, I. (1958). Selective cine-angiocardiography with image intensification. Brit. Heart J., 20, 459. 